\title{
THE SHRINKING AND EXPANDING HEURISTIC FOR THE FLEET SIZE AND MIX VEHICLE ROUTING PROBLEM
}

The FSMVRP (Fleet Size and Mix Vehicle Routing Problem) is a variant of the classical Capacitated Vehicle Routing Problem, CVRP. We suggest a new methodology, called the Shrinking and Expanding Heuristic (SEH) which is incorporated in a standard tabu search. To determine an appropriate fleet mix is a major challenge in this type of problem and the SEH technique is especially developed to find a good combination of vehicles by introducing a mechanism for changing the existing fleet mix during the search, thus also changing the underlying route structure. The SEH utilizes the concept of depletion and expansion of routes depending upon the filling degree of a vehicle. This strategy is tested on standard problem instances and good quality solutions are obtained.

Keywords: Shrinking and expanding heuristics, filling degree, fleet size and mix vehicle routing problem, tabu search.

\section{Introduction}

Distribution planning is a multifaceted field with a growing complexity in the decision making, involving fleet composition and routing decisions. Due to globalization and economic growth, distribution management and planning operations have become one of the key industrial advantages, in which optimization based approaches are expected to play a significant role. It shows that making routing decisions is challenging and crucial for the transportation planners and gives rise to tough competition among the transportation companies. The objective of transporters is to minimize the overall cost by visiting all of the customers in such a way that the transportation cost should be minimized. In general one assumes that the transportation cost accounts for about $20 \%$ of the total cost of a product [1]. Consequently, effective vehicle routing becomes vital for the transportation providers. The classic VRP is usually considered as a homogeneous fleet size problem and many researchers have used these assumptions. Presently, strengthening of the assumptions such as limited number of vehicles, capacity limitations and an appropriate composition of the fleet are considered. Therefore, this area of research is still flourishing due to the large growth of vehicle types and the implementation of new types of constraints related to this type of real world problems.

The layout of this paper is as follows. Section 1 gives a brief introduction to the FSMVRP followed by a problem description in Section 2. Section 3 provides a literature review related to the FSMVRP. Section 4 presents details of our searching procedure. Section 5 describes search parameter tuning and presents the final results with the appropriate found parameters. Our conclusions are presented in Section 6.

\section{Problem Description}

The FSMVRP can be considered as a deterministic delivery problem. It is defined on an undirected graph $G=(V, E)$ where $V$ is set of nodes (customers) consisting of $V=\{0,1, \ldots, n]$, and $E$ is set of edges which connects these nodes. Vertex 0 is the depot and the rest of the set, defined as $V^{\prime}=V \backslash\{0\}$ corresponds to $n$ nodes with non-negative demand $q_{i}$. The demand at a node is fulfilled by supplying $q_{i}$ units from the depot. The demand at the depot is considered to be zero $\left(q_{0}=0\right)$.

An unlimited heterogeneous fleet of vehicles is available. This fleet of vehicles is composed of $K=\{1, \ldots, k\}$ different vehicle types, each with a different cost structure and capacity. $F_{k}$ is the fixed acquisition cost for vehicle type $k$. Edge $e_{i j}$ belongs to the set of edges $E$ and has an associated non-negative cost $c_{i j}$. A pair $R\left\{r_{m}\right.$, $k$ ] defines a route $R$ which tells route number $r_{m}$ with a vehicle of type $k$ assigned to it.

The following are the customary constraints used for the general capacitated VRP.

i) Each customer should be visited once by exactly one vehicle.

ii) The vehicle routes must originate and terminate in the depot.

iii) The vehicle capacity should not be exceeded.

iv) There is only one depot.

In addition to these constraints, the following assumptions are usually made for the FSMVRP.

v) A fixed vehicle acquisition costs are considered for each vehicle type, i.e. $F_{k}>0$.

\footnotetext{
* Urooj Pasha, Arild Hoff, Arne Lokketangen

Molde University College, Specialized University in Logistics, Molde, Norway, E-mail: urooj.pasha@himolde.no
} 
vi) The traveling costs $c_{i j}$ between the nodes are generally dependent on vehicle type $k$.

vii) The cost of traveling between two nodes using the same type of vehicle would always be equal, i.e. $c_{i j}^{k}=c_{j i}^{k}$ (symmetric case).

Some of the above given constraints are not necessarily true for real world problems, but are often assumed so in the literature.

\section{Short Literature Overview}

The FSMVRP deals with the minimization of total transportation costs. This includes the fixed fleet acquisition cost plus variable traveling costs for the vehicle routes from the depot to customers and back to the depot. The vehicle types usually vary in sizes \& capacities. Unlimited number of vehicles and time is usually available. The types of vehicles vary in loading capacity and vehicles of different types have different costs for using and owning it. Hiring can also be an option, usually with a higher usage cost.

The research on FSMVRP has received some attention in the last two to three decades because of its practical importance. It is still a center of interest for researchers for its emerging constraints for a real world problem. The methods for solving the classical VRP are adapted by the researchers to solve the FSMVRP by considering the similarities of these problem types. Several heuristics were created by Golden et al. [2], based on the savings heuristic of Clarke and Wright [3]. The adaptation of the generalised assignment heuristic of Fisher and Jaikumar [4] was used by Gheysens et al [5]. Salhi and Rand [6] developed a new heuristic based on route perturbation (RPERT). Osman and Salhi [7] improved the RPERT heuristic later. The tabu search based meta-heuristics proposed by Gendreau et al. [8] have been used for solving the FSMVRP and Brandao [9] has presented a method including the GENIUS algorithm of Gendreau et al. [10]. Lastly, Subramanian et al. [11] have produced new best results on the classical test instances for FSMVRP by using Iterated Local Search [12].

Exact methods can only be used to solve small size instances [13], and thus heuristic methods are recommended to get good quality solutions within reasonable time when instance size increases. In this paper, a tabu search [13] based meta-heuristic is used to solve the FSMVRP.

\section{Tabu Search Methodology}

Our solution method is based on tabu search [13] (TS). We assume a general knowledge by the reader of TS, and will in the following explain the problem specific details of our method.

\subsection{Initial Solution Generation}

Various constructive heuristics have been formulated for the standard VRP. One of the best known of the classical heuristics is the Savings Heuristic defined by Clarke and Wright [3] (C\&W). This algorithm is used to produce good and diverse initial solutions.

\section{- Modified Savings Heuristic Initial Solution}

A constructive savings heuristic based on $\mathrm{C} \& \mathrm{~W}$ is used to get an initial solution. The $\mathrm{C} \& \mathrm{~W}$ algorithm is modified for the FSMVRP by selecting a vehicle type randomly and then the customer who is furthest away from the depot and whose demand is less than the vehicle capacity is added. Then inclusion of the remaining customers is evaluated and the one who adds a minimum value to the objective function is added to the route. The procedure is repeated as long as the vehicle capacity is not exceeded, and then another randomly generated vehicle is selected and the same procedure is applied.

\section{- Random Route Generation Initial Solution}

An alternative way to create initial solutions is to distribute customers randomly to different routes defined in total capacity by a random selected vehicle type. This procedure gives very low quality initial solutions, but this solution structure makes it easier for a simple tabu search to identify a proper fleet mix than constructive heuristics [14].

\subsection{Neighborhoods}

\section{- Shift Neighborhood}

This neighborhood mechanism works by moving a customer from its current route $A$ to another route $B$. Then both route $A$ and route B are re-optimized using 2-opt neighborhood [15]. All possible shift moves are considered and the one leading to the best solution is chosen.

\section{- Swap Neighborhood}

In a swap neighborhood, two customers are exchanged between different routes. Both of these routes are then re-optimized using 2-opt, and the best move is chosen.

\section{- Combined Neighborhood}

This is the combination of the shift and swap neighborhoods, as shown in Fig. 1. All shift and swap moves are evaluated and the move leading to the best solution is selected.

In the example given below, nodes $\mathrm{A}, \mathrm{B}$ and $\mathrm{C}$ are on three different routes R1, R2, and R3. In a swap move, the nodes A and B are exchanged between R2 and R1 respectively. In a shift move node $\mathrm{C}$ is moved from R3 to R2. Either a shift or a swap move is selected depending on objective function value improvement.

\subsection{Tabu and Aspiration Criteria}

Customers are considered tabu if they have been recently involved in a shift or swap move. The customer will be kept tabu for the duration of the tabu tenure and cannot be part of a move until the end of the tenure. The aspiration criterion is that a move 


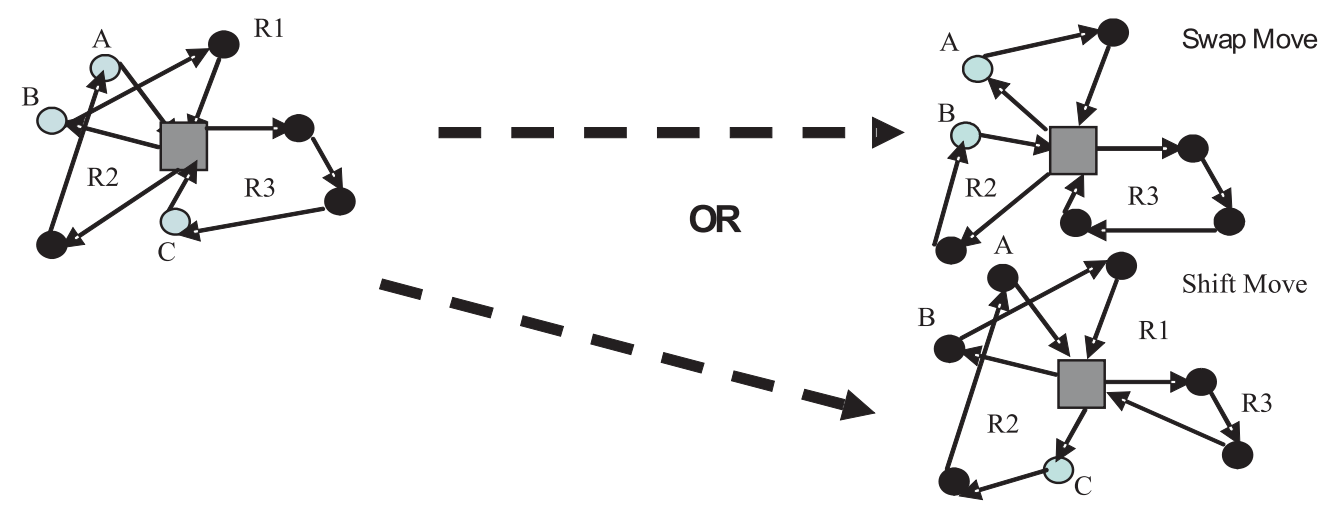

Fig. 1 Combined (Shift+Swap) Neighborhood

declared as tabu will be selected if it leads to a new best objective function value.

\subsection{Tabu Tenure}

This is the number of iterations that a customer is tabu. A variable tabu tenure $\theta$ is chosen as a random value within a given range. The values used are instance dependent, and $\theta$ is chosen as a random integer number in the interval between a lower and upper limit which are $l_{\min }$ and $l_{\max }$. These limits $l_{\min }$ and $l_{\max }$ (to be found while tuning parameters) are given as a percentage of the size of the instance, $n$.

\subsection{Penalty for Infeasible Solutions}

We allow the search to enter infeasible space with respect to violation of the overload constraint. A dynamic penalty factor $\beta$ is used to control the interplay between feasible and infeasible solutions and is explained in Fig. 3. The formula for adjusting the penalty factor is the same as used by Gendreau et al. [16]. ]. The overload is calculated for each of the routes and is multiplied by the penalty factor $\beta$ as given in Equation 1 whereas $p$ is the total penalty calculated for overload, $r$ is number of routes, $L_{r}$ represents the load on route $r$ and $Q_{r}$ is the capacity of the vehicle used by route $r$.

$$
p=\beta \cdot \sum_{1}^{r}\left(L_{r}-Q_{r}\right)
$$

\subsection{Termination Criteria}

The termination criterion to use in a search will usually depend on the time available. In our testing procedure, the termination criterion is set as a maximum iteration limit $\eta$ of iterations, where $\eta$ is a user controlled parameter.

\subsection{Shrinking and Expanding Heuristic (SEH)}

The selection of the right vehicle type to use for a route is hard. During the search, a move which allows a feasible assignment of a different vehicle type on a route will often be considered less attractive and therefore rarely chosen. Thus, to be able to change the fleet composition in a better/proper way during the search, some indications are needed to find out when this is favourable. One such indication is the filling degree (FD), defined as the percentage utilization of the vehicle capacity on a route. A route with an FD close to $100 \%$ will probably be a good route which should not be chosen for reduction or expansion. Other routes with a smaller FD (e.g. with half or two third filled vehicle) could however be candidates for a change to a smaller vehicle type for obtaining a better fleet mix. A threshold of the FD on a route could indicate whether an increase or decrease of the capacity on the route should be performed. In our heuristic, a route with an FD higher than the threshold but not too close to $100 \%$ could possibly be a good route for expansion. If such a route is already using the largest vehicle then that route would be split into two routes by finding two vehicles which adds extra capacity compared to the earlier used vehicle on the route. Similarly, if the FD on a particular route is smaller than the threshold then this could be an indication that the route can be served by a smaller vehicle or be completely depleted by assigning all the customers to other routes. This intuition is used for the implementation of the Shrinking and Expanding Heuristic (SEH). After a given number of iterations in the search without an improvement, this procedure is called, and a route which satisfies the filling degree criterion is selected. Depending on the filling degree criterion, the corresponding action is carried out. If a larger vehicle is assigned to the selected route then it will have extra capacity to add more customers. These are selected greedily from the neighboring routes and added to this route. If the filling degree is much smaller than the vehicle capacity, then the route is selected for depletion. Customers are removed from the selected route and added greedily to the neighboring routes, adjusting the vehicles on those routes accordingly. A smaller vehicle is assigned to the reduced route, or if all customers are removed, the route is depleted. This case is illustrated in Fig. 2. 

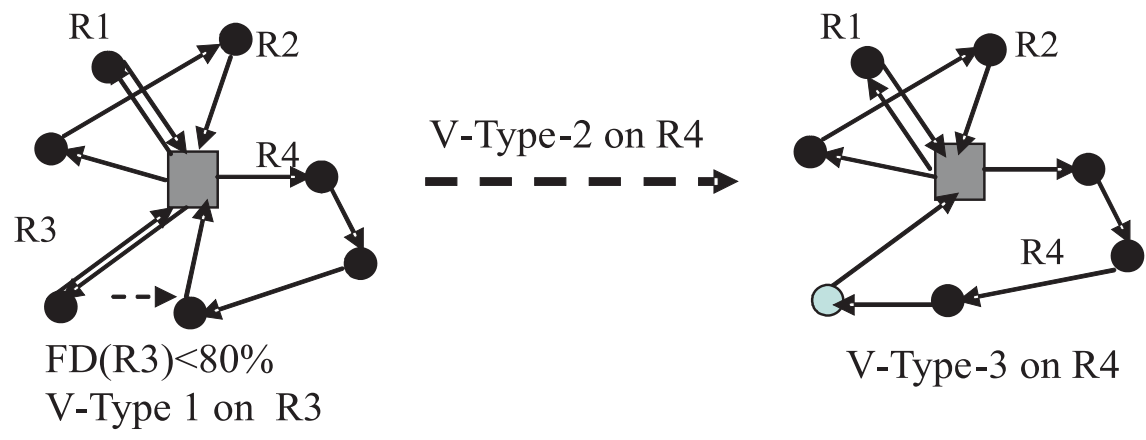

Fig. 2 Shrinking and Expanding Heuristic for changing vehicles

If there is a slack on route R4 with vehicle V-Type-2, this route $\mathrm{R} 4$ will be selected for expansion depending on filling degree. A customer is shifted from route R3 to route R4 and R4 will be expanded. The route $\mathrm{R} 3$ will be discarded if it has no customers. The larger vehicle V-Type-3 is assigned to R4 as shown in Fig. 2.

\subsection{The Tabu Search Algorithm}

The description of the tabu search algorithm with the variables and parameters used is presented in Fig. 3.

$s_{0}$ Initial solution

$s_{b}$ Best solution

$s_{c} \quad$ Current solution

$\eta \quad$ Total number of iterations

$a \quad$ Adjustment factor for $\beta$

$\beta \quad$ Penalty factor for overload $c(s)$ Total routing cost of selected solution

$\tau$ Tabu list

$\theta \quad$ Tabu tenure

$\rho \quad$ Frequency of calling SEH

\section{Parameter Tuning and Computational Results}

Our solver is coded in C++ using Microsoft Visual Studio 2007 running on Intel Pentium(R)-IV machines with a $2.4 \mathrm{GHz}$ processor and using the Microsoft Windows XP operating system.

\subsection{Test Data.}

The common benchmark instances for FSMVRP used by Golden et al. [2] are used to test the quality of different heuristics.

1. Initialization

Generate initial solution $s_{0}$

Set $s_{c}:=s_{0}$

2. Initialize all parameters and variables $\theta, \tau . \rho . \beta . \eta$. $\alpha$.

3. Tabu search

Repeat iterations $\eta$ times

a) Check all solutions in the selected neighborhoods

b) Select sc as the best non-tabu solution in the neighborhoods

c) If a tabu solution fulfills the aspiration criterion, set this solution as $s_{c}$

d) Update tabu status $\tau$, tabu tenure $\theta$

e) Update penalty factor for infeasible solutions

$$
\begin{aligned}
& \text { if sc is infeasible } \\
& \quad \beta=\beta^{*}(1+\alpha) \\
& \text { else } \\
& \quad \beta=\beta^{*}(1-\alpha)
\end{aligned}
$$

f) if $s_{c}$ is feasible and $c\left(s_{c}\right)<c\left(s_{b}\right)$

save $s_{b}=s_{c}$

j) if $\rho$ iterations without improvement $s_{b}$ call SEH procedure

4. Report $s_{b}$ and stop

Fig. 3 Tabu search algorithm using SEH 
These test instances are divided into two sets ranging from 12 to 100 customers. One set explicitly defines the distance between the nodes (i.e. it is assumed that distance unit is equal to the cost unit and a node is defined as a customer), and the other set specifies the coordinates of the nodes and uses the Euclidean distance between the nodes. The test instances contain data about the depot node, available vehicle types with different costs and capacities, and, the cost assigned for traveling between the nodes and the customer node's demand. For these instances, the vehicle types are not homogeneous.

\subsection{Tuning of parameters.}

To be able to utilize a meta-heuristic in the best possible way, a careful tuning of the search parameters is necessary. Testing for parameter setting has been performed on a subset of four of the test instances. These test instances are chosen from the same benchmark set as used for general testing. They are selected on the basis of vehicle types, size of problem and its type. Two problems have exact distances between the customers and include twelve and thirty customers respectively. Two other problems use Euclidian distances between the customers and include twenty and fifty customers respectively. Tests for parameter settings are run several times with twenty different random seed values. The sample test instances named as $1 \mathrm{~S}, 5 \mathrm{E}, 12 \mathrm{~S}$ and $15 \mathrm{E}$ are taken from Golden et al. [2].

The preliminary tests result indicates that using variable tabu tenure between $10-35 \%$ of the instance size in terms of number of customers gives best results. Standard TS with or without SEH is tried with initial solutions using both the random route generation and the modified savings heuristics. Our testing shows that when the search is limited to only feasible solutions a randomly generated initial solution gives better results, but when the infeasible space is included in the search, an initial solution found by the savings heuristic leads to better overall solutions using SEH technique.

\subsection{Parameters Tuning for the Shrinking and Expanding Heuristic}

The SEH strategy is developed to check the FD for each route during the tabu search when it has not improved for a predefined number of iterations. Thus, to utilize the strategy, a decision on how to use the FD to select which route to change the vehicle size needs to be taken. Another difficulty is the selection of a threshold for the FD. This heuristic performs several shift moves in a sequence to fill up a larger vehicle on a route or to reduce/empty a route enough to be able to use a smaller vehicle. Customers chosen for shift moves are selected on the basis of two characteristics, i.e. the closest customer(s) to the current route and whose demand is less than or equal to available slack on the current route. Thus, the following parameter values need to be decided:

- Which route should be chosen for a vehicle change when the SEH procedure is called?
- Which threshold of filling degree should indicate whether to increase or decrease the vehicle type?

- How often should the SEH procedure be called?

Our preliminary tests showed that routes with a higher FD than $96 \%$ should not be changed. A route was chosen at random among the others and a threshold value of $80 \%$ was favorable for deciding whether to increase or decrease the vehicle capacity on the route. Finally, calling the SEH procedure after 30 iterations without improvement in the search turned out to be the frequency giving best results.

\subsection{Comparison of Standard Tabu Search with and without the Shrinking \& Expanding Heuristic}

The performance of a tabu search with the Shrinking and Expanding Heuristic is compared with a tabu search not using this technique. Both searching only in feasible space and searching in both feasible and infeasible space. Results calculated by comparing the average deviation from the best known solutions (BKS) for the selected instances are shown in Figs. 4 and 5.

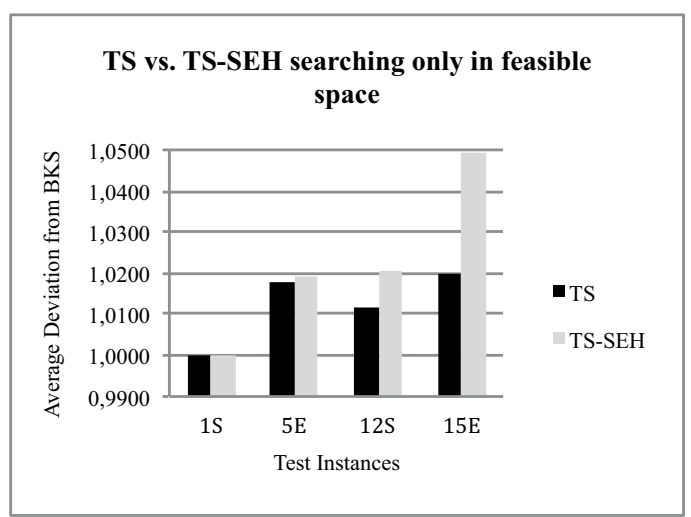

Fig. 4 Average deviation for a TS-SEH on four test problem instances searching in feasible space only.

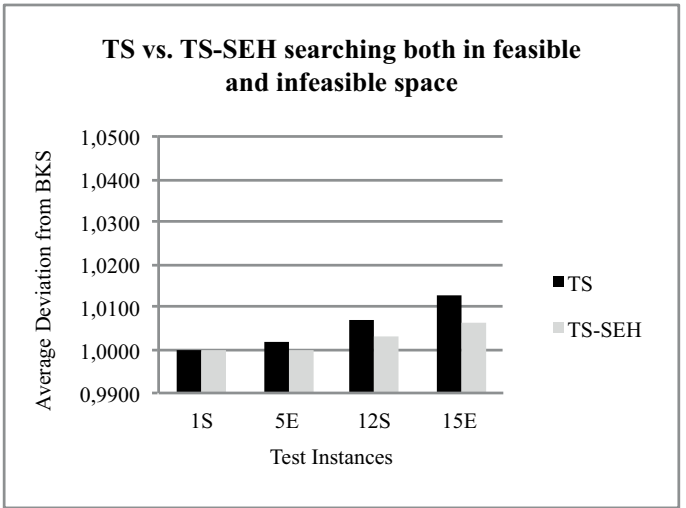

Fig. 5 Average deviation for a TS-SEH on four test problem instances searching in feasible and infeasible space. 


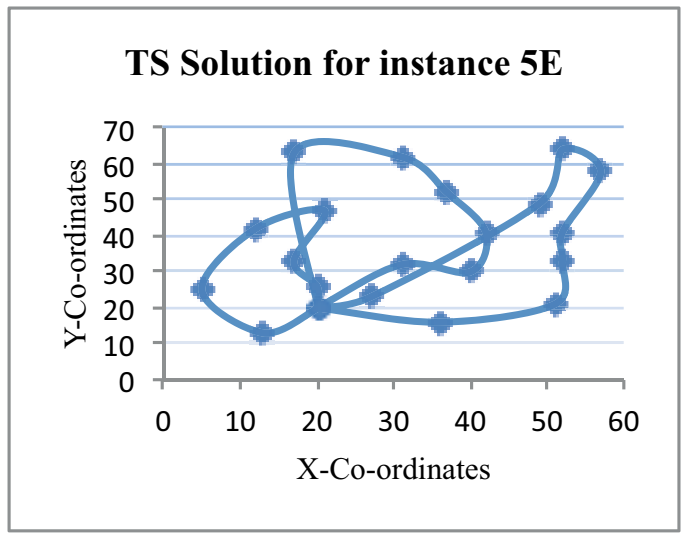

\section{TS-SEH Solution for instance $5 E$}

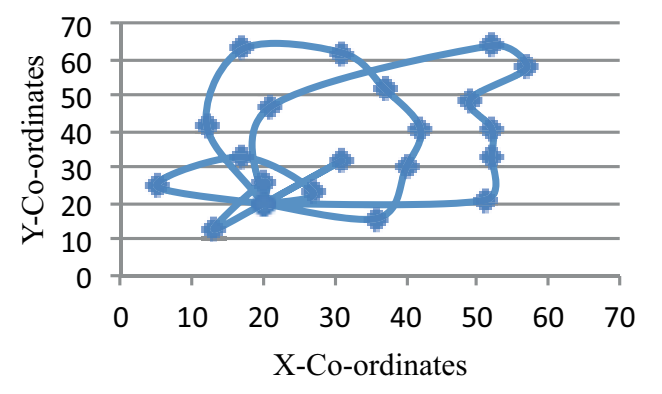

Fig. 6 Graphical solution found by a standard TS and TS-SEH for test instance 5E.

It can be seen from Fig. 4 that searching in feasible space only, may yield reasonably good solutions, but the SEH strategy does not seem to improve solutions compared to a search without this strategy. Due to the limited search space, it seems difficult to improve after a good solution is found. However, if the search is allowed to go into infeasible space, it would improve the solutions found by searching only in feasible space considerably, and the benefit of the SEH technique is clearly seen in Fig. 5.

\subsection{Graphical Representation of a FSMVRP-Solution}

The instance 5E from [2] with Euclidian distances is chosen to show the effect of the SEH strategy and presented graphically in Fig. 6.The tabu search not using the SEH technique performed well, but the result is slightly poorer than the result when including this technique.

The cost of the solution found by standard tabu search is 1008.59 and it is using the largest vehicle type on all three routes, as can be seen in Fig. 6. The TS-SEH search however, is able to identify the diverse fleet composition and routing which is known as the best solution from the literature, in which the cost is 1007.05 . Even if the difference between the solutions is very nominal, the TS-SEH shows its advantage in finding the best fleet mix among the available vehicles.

\subsection{Final Computation}

The parameter tuning for the tabu search algorithm is explained in Section 5.2 and 5.3 and the values and strategies shown below are used for the final experiments. The same version of the TS-SEH with two different initial solutions was tested with 20 different seeding values. The best results found are selected and presented in the final results. The values of parameters used for the final implementation of the algorithm are given in Table 1.
The initialization of parameters for final computation

Table 1 of TS-SEH algorithm

\begin{tabular}{|c|c|c|}
\hline Parameters & Notation & Initial values \\
\hline Tabu Tenure & $\theta$ & $\begin{array}{c}\text { Randomly between } 10-35 \% \text { of } \\
\text { instance size }\end{array}$ \\
\hline NH structure & $\kappa$ & Combined \\
\hline Frequency Call & $\rho$ & 30 \\
\hline Penalty factor & $\beta$ & random [0.1-0.9] \\
\hline Adjustment Factor & $\alpha$ & random [0.01-0.09] \\
\hline Filling degree & $\delta$ & $80 \%$ \\
\hline Termination criterion & $\eta$ & 4000 \\
\hline
\end{tabular}

\subsection{Comparison with other researchers}

The best results found using the presented algorithm are compared with those given by other researchers and presented in Table 2. The last column presents the results found by using our methodology.

The column names are explained in Table 3 with solution methodology implemented by each of these researchers and mentions the references accordingly.

The methodology implemented in this research paper is a tabu search heuristic with the SEH technique for better identifying the optimal fleet mix. The tabu search uses a simple neighborhood structure without any diversification and intensification mechanisms. Still, the results are competitive with the results found by other researchers on standard test instances. Table 2 shows that our search performs better than constructive heuristics such as Golden et al. [2], but not as good as more advanced tabu search heuristics recently developed by Brandao [9] and Subramanian et al. [11]. 
Comparison of results

Table 2

\begin{tabular}{|c|c|c|c|c|c|c|c|}
\hline Problem instance & Best known solution & GALG & OS & GLMT & B & SPOU & $P H L$ \\
\hline $1 S$ & 602.00 & 622.00 & 602.00 & - & 602.00 & - & 602.00 \\
\hline SS & 722.00 & 722.00 & 722.00 & - & 722.00 & - & 722.00 \\
\hline 3E & 961.03 & 966.00 & 971.24 & 961.03 & 961.03 & 961.03 & 961.92 \\
\hline $4 \mathrm{E}$ & 6437.33 & 6930.00 & 6445.10 & 6437.33 & 6437.33 & 6437.33 & 6437.33 \\
\hline 5E & 1007.05 & 1013.00 & 1009.15 & 1007.05 & 1007.05 & 1007.05 & 1007.05 \\
\hline $6 \mathrm{E}$ & 6516.47 & 6974.00 & 6516.56 & 6516.46 & 6516.47 & 6516.47 & 6516.47 \\
\hline $7 \mathrm{~S}$ & 7273.00 & 7389.00 & 7310.00 & - & 7273.00 & - & 7285.00 \\
\hline $8 \mathrm{~S}$ & 2346.00 & 2367.00 & 2348.00 & - & 2347.00 & - & 2347.00 \\
\hline $9 \mathrm{~S}$ & 2209.00 & 2220.00 & 2209.00 & - & 2209.00 & - & 2209.00 \\
\hline $10 \mathrm{~S}$ & 2355.00 & 2370.00 & 2363.00 & - & 2355.00 & - & 2355.00 \\
\hline $11 \mathrm{~S}$ & 4755.00 & 4763.00 & 4755.00 & - & 4755.00 & - & 4755.00 \\
\hline $12 \mathrm{~S}$ & 4080.00 & 4136.00 & 4092.00 & - & 4080.00 & - & 4092.00 \\
\hline $13 \mathrm{E}$ & 2406.36 & 2438.00 & 2471.07 & 2408.41 & 2406.36 & 2406.36 & 2462.63 \\
\hline $14 \mathrm{E}$ & 9119.03 & 9132.00 & 9125.65 & 9119.03 & 9119.03 & 9119.03 & 9153.63 \\
\hline $15 \mathrm{E}$ & 2586.37 & 2640.00 & 2606.72 & 2586.37 & 2586.37 & 2586.37 & 2603.33 \\
\hline $16 \mathrm{E}$ & 2720.43 & 2822.00 & 2745.01 & 2741.50 & 2728.14 & 2720.43 & 2742.52 \\
\hline $17 \mathrm{E}$ & 1734.53 & 1783.00 & 1762.05 & 1749.50 & 1734.53 & 1734.53 & 1744.45 \\
\hline $18 \mathrm{E}$ & 2369.65 & 2432.00 & 2412.56 & 2381.43 & 2369.65 & 2369.65 & 2439.90 \\
\hline $19 \mathrm{E}$ & 8661.81 & 8721.00 & 8685.71 & 8675.16 & 8661.81 & 8661.81 & 8668.63 \\
\hline $20 \mathrm{E}$ & 4032.81 & 4195.00 & 4188.73 & 4086.76 & 4042.59 & 4032.81 & 4092.67 \\
\hline Average Deviation & 1.0208 & 1.0073 & 1.0046 & 1.0003 & 1.0000 & 1.0049 \\
\hline
\end{tabular}

Solution Methodologies by researchers

\begin{tabular}{|l|l|l|}
\hline Col. name & Author(s) & Solution methodology \\
\hline GALG & Golden et al. [2] & Giant Tour \\
\hline OS & Osman and Salhi [7] & Modified Route Perturbation \\
\hline GLMT & Gendreau et al. [8] & GENIUS - Tabu Search \\
\hline B & Brandao [9] & Tabu Search \\
\hline SPOU & Subramanian et al. [11] & ILS based on Set Partitioning \\
\hline PHL & Pasha et al. & TS-SEH \\
\hline
\end{tabular}

By not using the SEH strategy, the tabu search will in general lead to fewer routes with larger vehicles as it can be seen in Fig. 6 . The solver is executing the shift and swap neighborhoods which
Table 3 is creating a large neighborhood when the size of the instance is large, which in turn will increase the searching time.

Our method has reproduced eight of the best known solutions on the test instances and overall the solutions found are within $1 \%$ deviation from the best known solutions on all 20 test instances.

\section{Conclusion}

In this research, a tabu search algorithm with a simple neighborhood and a new technique for identifying the fleet mix has been implemented. The parameter values were selected carefully by thorough testing on a subset of the problem instances used.

One advantage of using the shrinking and expanding technique is that it makes the search more robust and reduces the randomness and variance of the search.

\section{References}

[1] HOFF, A., ANDERSSON, H., CHRISTIANSEN, M., HASLE, G., LOKKETANGEN, A.: Industrial Aspects and Literature Survey: Fleet Composition and Routing. Computers \& Operations Research 37, 2010, pp. 2041-2061. 
[2] GOLDEN, B., ASSAD, A., LEVY, L., GHEYSENS, F.: The Fleet Size and Mix Vehicle Routing Problem. Computers \& Operations Research 11, 1984, pp. 49-66.

[3] CLARKE, G., WRIGHT, J. V.: Scheduling of Vehicles from a Central Depot to a Number of Delivery Points. Operations Research 12, 1964, pp. 568-581.

[4] FISHER, M. L., JAIKUMAR, R.: A Generalized Assignment Heuristic for Vehicle Routing. Networks 11, 1981, pp. 109-124.

[5] GHEYSENS, F., GOLDEN, B., ASSAD, A.: A Comparison of Techniques for Solving the Fleet Size and Mix Vehicle Routing Problem. Computers \& Operations research 11 (1), 1986, pp.49-66.

[6] SALHI, S., RAND, G. K.: Incorporating Vehicle Routing into the Vehicle Fleet Composition Problem. European J. of Operations Research 66, 1993, pp. 313-330.

[7] OSMAN, I., SALHI, S.: Local Search Strategies for the Vehicle Fleet Mix Problem. In: RAYWARD-SMITH, V. J., OSMAN, I. H., REEVES, C. R., SMITH, G. D. (Eds), Modern Heuristic Search Methods. Wiley : New York, 1996, pp.131-153.

[8] GENDREAU, M., LAPORTE, G., MUSARAGANYI, C., TAILlARD, E. D.: A Tabu Search Heuristic for the Heterogeneous Fleet Vehicle Routing Problem. Computers \& Operations Research 26, 1999, pp. 1153-1173.

[9] BRANDAO, J.: A Deterministic Tabu Search Algorithm for the Fleet Size and Mix Vehicle Routing Problem. European J. of Operational Research 195, 2009, pp. 716-728.

[10] GENDREAU, M., HERTZ, A., LAPORTE, G.: New Insertion and Post-Optimization Procedures for the Traveling Salesman Problem. Operations Research 40 (6), 1992, pp. 1086-1094.

[11] SUBRAMANIAN, A., PENNA, P. H. V., UCHOA, E., OCHI, L. S.: A Hybrid Algorithm for the Heterogeneous Fleet Vehicle Routing Problem. European J. of operational research 221, 2012, pp. 285-295.

[12] LOURENCO, H. R., MARTIN, O., STUTZLE, T.: Iterated Local Search. In GLOVER, F., KOCHENBERGER, G., (eds.), Handbook of Metaheuristics, Kluwer Academic Publishers, 2003, pp. 321-353.

[13] GLOVER, F., LAGUNA, M.: Tabu Search. Kluwer Academic Publishers, 1997.

[14] PASHA, U.: A Tabu Search Heuristic for Solving the Fleet Size and Mix Vehicle Routing Problem. Master thesis, Molde University College : Molde, 2008.

[15] FLOOD M. M.: The Traveling Salesman Problem. Operations Research 4, 1956, pp. 61-75.

[16] GENDREAU, M., HERTZ, A., LAPORTE, G.: A Tabu Search Heuristic for the Vehicle Routing Problem. Management Science 40, 1994, pp. 1276-1290. 